\title{
Research on the High-power Directional Acoustic Transducer
}

\author{
ZHAO Guo Zhu1, a, CHEN You Jun ${ }^{2, b}$ \\ ${ }^{1}$ Civil Aviation Flight University of China Guanghan Sichuan, China \\ ${ }^{2}$ Civil Aviation Flight University of China Guanghan Sichuan, China \\ a jude.1909@163.com, b379175094@qq.com
}

\begin{abstract}
Keywords: Directional audio, Transducer, Impedance analysis, Class-D amplifier, Directivity pattern.

Abstract. Through studying how to affect acoustic directivity with MATLAB software, it show that selection of a relatively larger surface of the transducer and a relatively closer transducer interval will be more preferable for directivity. While an array which possess more array element number and whose frequency of the drive signal can be as large as possible in a range, directivity will be more preferable. On the other hand, when the structure of the sound radiating surface of the transducer or array layout is symmetrical, the corresponding directivity pattern will be symmetrical. At a frequency of $10 \mathrm{kHz}$, the individual rare earth magnetostrictive transducers its first point of the simulation carried out before the test. Sounding board with aluminum by the method used to improve the sound source diameter. With the sound plate diameter incrementing, the smaller the angle of the directivity. The beam width of the $3 \times 3$ array is about $\Theta_{-3 d B} \leq 20$ at $23 \mathrm{kHz}$, and the directivity acute angle is about $10^{\circ}$, with a sounding board by the method used to improve their the diameter of the sound source, so as to realize the sound has directivity is feasible.
\end{abstract}

\section{Introduction}

Magnetostrictive implies that the material stretching across the magnetic field will occur. Specifically, the material is magnetized in a magnetic field when an electric signal by the action occurs in the magnetization direction of elongation or shortening effect. Magnetostrictive material because of its common strain is small, the coupling difficulties and other reasons have been unable to produce high-power ultrasonic transducer, can not achieve large-scale applications. In recent years, people are exploring a new and high electromechanical conversion efficiency, and can be used to design high power transducer magnetostrictive material, which is called the super magnetostrictive material ${ }^{[1]}$.

Magnetostrictive coefficient " $\lambda$ " is generally used to indicate the size of the magnetostrictive effect, the unit is a small unit, on behalf of millionths of a meter. Strain traditional magnetic magnetostrictive material is very small, limiting their potential applications. $\Lambda$ value of the new super-magnetostrictive material is large, compared with traditional materials to two orders of magnitude or more. From traditional materials of $10 \sim 100 \mathrm{ppm}$ up to 1000 2500ppm, which is a breakthrough ${ }^{[2,3,4]}$.

\section{Directional sound pressure meter testing with Matlab}

Magnetic super-magnetostrictive material electrostrictive value, electromechanical coupling factor, energy density and other parameters are much larger than conventional magnetostrictive material. This greatly broadens the practical applications. Can be used in sonar systems, high-power ultrasonic devices, ultrasonic flaw detection systems, precision control systems, high-energy micro-power source, and a variety of sensors, and so on. Orientation is also an ideal material for the production of the acoustic transducer. This kind of super-magnetostrictive material with incomparable advantages of piezoelectric ceramic materials, especially for high-power applications. It has high mechanical strength, without causing damage when the power system; super magnetostrictive material does not exist in the piezoelectric ceramic will produce an electric field produced by a residual polarization may be degraded over time and eventually produce polarization problem. 
For high-power piezoelectric transducers, piezoelectric materials may produce permanent depolarization, in this situation, even momentary overload may also cause depolarization phenomenon is more obvious, and even the giant magnetostrictive material is heated to a that does not produce adverse reactions when the Curie point of the piezoelectric only transient stretching. When the temperature drops can be restored, indicating that this material is very stable and reliable performance. From the mid-1970s, GMM research focuses on the impact of each material composition and preparation of materials for its performance, accelerate the practical use of technology and products. To around 90 years, a number of countries to achieve the industrialization GMM production, such as the U.S. Etrema's Terfenol-D and Sweden in a company Magmek86, then including China, Britain, Japan, Russia and other countries have also developed a $\mathrm{TbDyFe}_{2}$ type GMM rod ${ }^{[5,6,7]}$.

Our research began in the 2011about the material, there are a number of units and production GMM rod, such as Gansu Province Star Gansu Rare Earth Functional Materials Co., Ltd., Baotou Rare Earth Research Institute, Beijing Nonferrous Metal Research Institute, Institute of Physics, Zhejiang pepper light rare earth materials Co., Ltd., and its main performance indicators are approaching or have reached the international advanced level of similar products.

$$
\begin{aligned}
f(t) & =\sum_{i} a_{i} \sin \omega_{i} t \\
f^{\prime}(t) & =\left(\sum_{i} a_{i} \sin \omega_{i} t\right)^{2} \\
f^{\prime}(t) & =\sum b_{i, j}\left[\cos \left(\omega_{i}+\omega_{j}\right) t-\cos \left(\omega_{i}-\omega_{j}\right) t\right]
\end{aligned}
$$

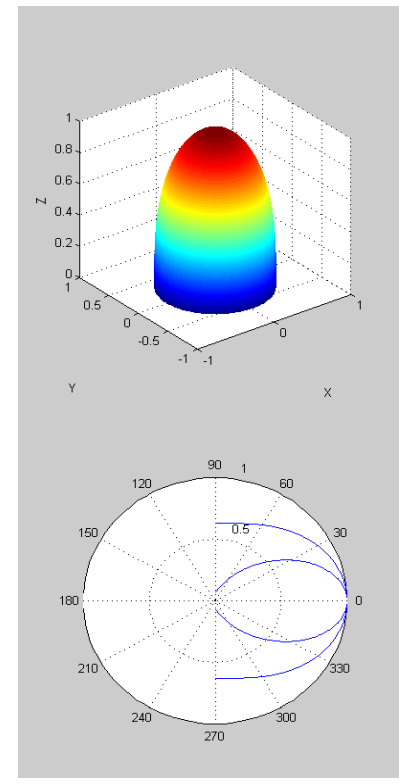

a $\mathrm{d}=38 \mathrm{~mm}$

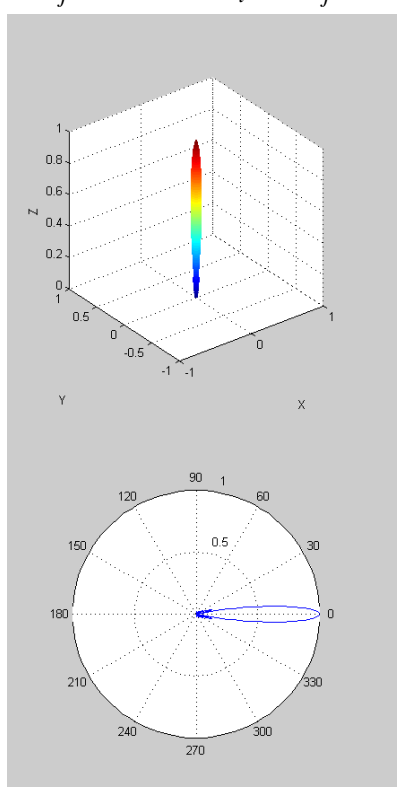

b d $=200 \mathrm{~mm}$

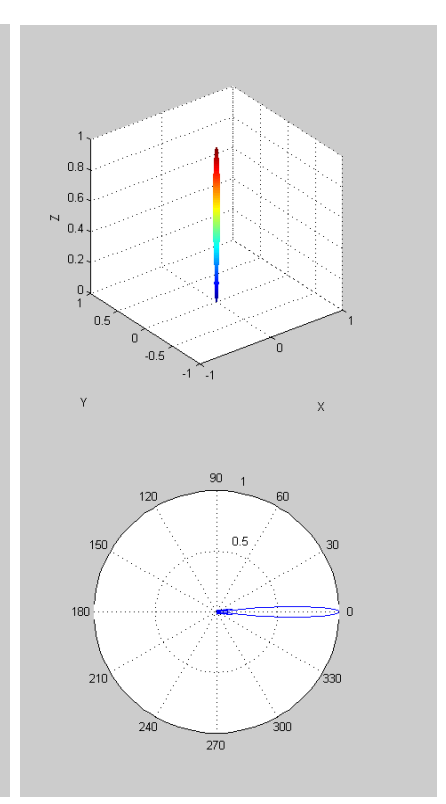

c $\mathrm{d}=300 \mathrm{~mm}$

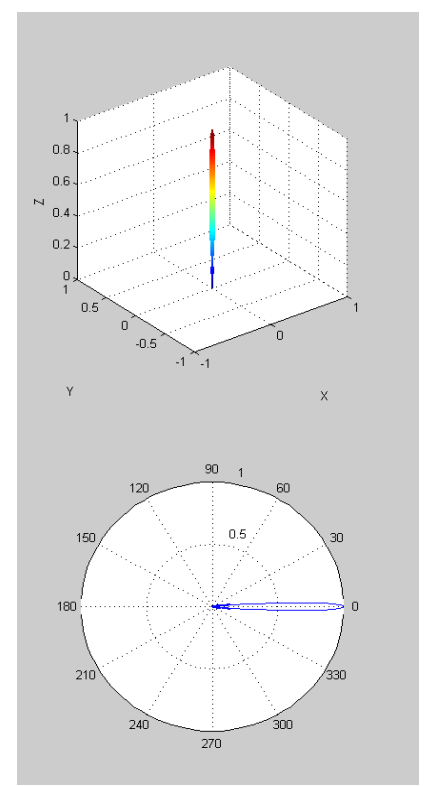

$\mathrm{d} \mathrm{d}=400 \mathrm{~mm}$

Figure 1. sound transducers with different directivity diagram plate

Table 1 occurred plate diameter and directivity angle relationship

\begin{tabular}{|l|l|l|}
\hline diameter & angle & effect \\
\hline $38 \mathrm{~mm}$ & $54.2714^{\circ}$ & Less is better \\
\hline $200 \mathrm{~mm}$ & $9.9497^{\circ}$ & better \\
\hline $300 \mathrm{~mm}$ & $7.2362^{\circ}$ & better \\
\hline $400 \mathrm{~mm}$ & $5.4271^{\circ}$ & better \\
\hline
\end{tabular}

At a frequency of $10 \mathrm{kHz}$, the individual rare earth magnetostrictive transducers its first point of the simulation carried out before the test, the simulation results shown in Figure 1. Sounding board with aluminum by the method used to improve the sound source diameter. As can be seen in Table 1, with the sound plate diameter (denoted by d) incrementing, the smaller the angle of the directivity, that: at 
the frequency of the frequency at $10 \mathrm{kHz}$, with a sounding board by the method used to improve their the diameter of the sound source, so as to realize the sound has directivity is feasible.

Since this kind of transducer higher costs, development costs of complex reasons, not the kind of temporary transducer array consisting of verification.

\section{Test directionality with sound pressure}

The dicrectionality is that at a certain frequency, the transducer response is used as a function of acoustic emission or incoming sound waves through the specified plane of the sound center. Directivity acute Angle is that the directive Angle is the Angle of the direction of the main propagating axis in the direction of the main propagation axis, and the Angle of the direction is half of the directional Angle. Most of the noise sources have certain directionality. Under a given frequency band, a fixed distance from the sound source, when the sound pressure level of the sound source radiation is measured, it is often found that the sound pressure is different in different directions, in some parts of life experience is sound "big", "small" voice somewhere. So there is the concept of "directionality", which is now commonly used to describe the variation of sound pressure levels in different directions at a certain distance.

\section{Conclusions}

As shown in Figure 2 is a magnetostrictive transducer and piezoelectric transducer operating range comparison chart, piezoelectric transducers are commonly used ultrasonic frequency, greater than $20 \mathrm{kHz}$, and magnetostrictive transducers can be applied to both acoustic wave, but also can be applied to ultrasound.

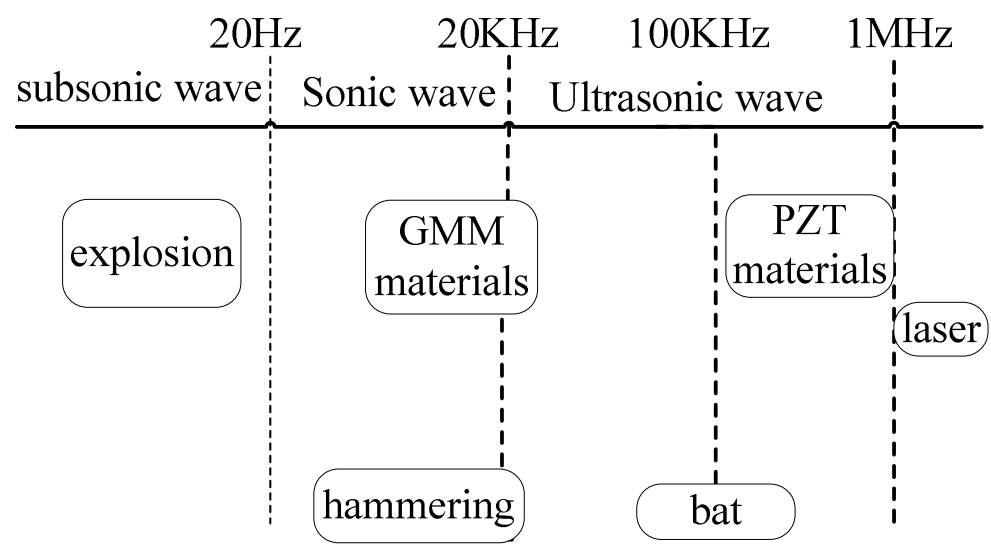

Figure 2. giant magnetostrictive material and piezoelectric material scope comparison chart

For this problem, the transducer selected basic requirements are: the operating frequency greater than $20 \mathrm{kHz}$, and air can be preferably coupled for ultrasonic waves in air, the sound pressure level to be as large as possible. For an array consisting of a plurality of transducers, in order to make the array can be synchronized using the series-parallel arrays, generally do not have more than one power amplifier are to each transducer, the reason is the high cost of multiple amplifier design and a plurality of power amplifiers work alone can not maintain the consistency of the signal. As the series plurality of transducer failure may occur resulting in a single transducer transducer array fail result, the final design is parallel transducer array.

The graph compares the obtained impedance, and the speaker can not find common resonance frequency, and the selected piezoelectric transducer having a resonant frequency significantly, through repeated testing and observation, the resonant frequency is found at about $23 \mathrm{kHz}$ and $47 \mathrm{kHz}$. However, the gap due to the design or production process, not all of the transducers are the frequency, but in the vicinity of these two frequencies, but through a large number of tests and found that the selected group of transducers better consistency ${ }^{[8-11]}$.

By comparison, select the matching effect, a simple circuit structure of the series inductive impedance matching circuit as the final circuit. Since the inductance of the inductor $1.02 \mathrm{mH}$ not 
readily available, the final choice $1 \mathrm{mH}$ inductor as the selected components, and transducers form a series circuit, in which the latter part of the experiment, the actual test is better.

Magnetic finite element model can be seen, Terfenol-D has a significant magnetic field distribution in the Terfenol-D, the magnetic field is strongest. At a frequency of $10 \mathrm{kHz}$, with a sounding board by the method used to improve the magnetic quality sound transducer diameter telescopic achieve its sound directivity is feasible.

In subsequent chapters, directional acoustic transducer circuit platform designed primarily uses a piezoelectric transducer as a basic component, tested and validated on this basis. Quality telescopic magnetic transducer validation work will be carried out in subsequent research work, this paper because of technical and cost aspects temporarily for testing.

\section{Acknowledgements}

This work was financially supported by fund project of Civil Aviation Flight University of China (Q2018-170) and (J2015-63). Helicopter multi mission flight training device development, civil aviation innovation and guidance fund projects major projects ( MHRD20130108 ).

\section{References}

[1] Chen Min. Research on the Fundamental Theory and Key Technologies of Audio Directional System [PhD]. University of Electronic Science and Technology of China, (2008).

[2] Masashi Yamada, Nobuyuki Itsuki, Yohsuke Kinouchi. Adaptive Directivity Control of Speaker Array[J]. Proceedings of the 8th International Conference on Control, Automation, Robotics and Vision, Kunming, China, Dec 6-9, (2004).

[3] M. Yoneyama, J.-I. Fujimoto. The Audio Spotlight: An Application of Nonlinear Interaction of Sound Waves to a New Type of Loudspeaker Design[J]. The Journal of the Acoustical Society of America, (1983).

[4] Woon-Seng Gan, Jun Yang, Khim-Sia Tan, et al. A Digital Beamsteerer for Difference Frequency in a Parametric Array[J]. IEEE Transactions on Audio, Speech and Language Processing(2006).

[5] T. Kamakura, T. Yoneyama and K. Ikegaya. Studies for the Realization of Parametric Loudspeaker[J]. Journal of Acoustic Society of Japan(1985).

[6] T. Kamakura, M. Yoneyama, K. Ikegaya. Developments of Parametric Loudspeaker for Practical Use[J]. 10th International Symposium on Nonlinear Acoustics, Kobe, Japan(1984).

[7] F. J. Pompei. The Use of Airborne Ultrasonics for Generating Audible Sound Beams[J]. Journal of Audio Engineering Society(1999).

[8] J. James, J. O. Norris. HSS white paper[M]. USA: American Technology Corporation(2005).

[9] Dekun Yang, Stuart Flockton. An Evolutionary Algorithm for Parametric Array Signal Processing[J]. Lecture Notes in Computer Science(1995).

[10] Furi Andi Karnapi, Woon Seng Gan, Y.K.Chong. FPGA Implementation of Parametric Loudspeaker System[J]. Microprocessors and Microsystems(2004).

[11] M. Zheng, R.F.W. Coates. Angular Response of a Parametric Array: Analytical Solution[J]. Journal of Sound and Vibration(1998). 